\title{
An observational study on the long term follow-up following thermablate and novasure endometrial ablations
}

\begin{abstract}
Objectives: A 5-year observation study investigated the quality of life and intervention rates following the Thermablate thermal balloon endometrial ablation (Thermablate EA) and the NovaSure radiofrequency impedance-based ablation (Novasure).

Methods: Anonymous information for the study was gathered from 'NOTIS', the electronic system used within our Trust to hold patient data. Following the ablation subsequent procedures that occurred were listed as well as the bleeding pattern recorded.

Results: There were 123 women treated with the Novasure system and 168 women with Thermablate EA. A greater number of women treated with the Novasure system became amenorrheic (64\%) compared to the Thermablate EA group (24\%). However more women who were treated with the Novasure system compared to the Thermablate EA suffered post ablation pain, (13.8\% and 1.8\%) and remained unsatisfied. In the Novasure group, 67.8 $\%$ had no subsequent interventions as compared to $75.6 \%$ in the Thermablate EA group $(\mathrm{p}=.001)$. In the Thermablate EA group, $7 \%$ had subsequent medical treatment compared to $12 \%$ of the NEA women. There are more women opted for hysterectomy in the Novasure group $(20.3 \%)$ as compared to $8.3 \%$ in the Thermablate EA. The odds ratio of women having hysterectomy after the Novasure as compared to Thermablate EAS group is 2.8 $(95 \% \mathrm{CI}=1.37-5.71)$.

Conclusion: Women may prefer the Thermablate EA with the associated less medical intervention and hysterectomy rates despite more women becoming amenorrheic after a Novasure treatment. This has implications on cost and patient satisfaction when deciding on initial treatment.
\end{abstract}

Keywords: NovaSure, Thermablate, Endometrial ablation, Hysterectomy, Intervention rate, Thermal balloon
Volume 7 Issue 3 - 2017

\author{
Bee Fong Chen, Rozmina Mohamed, Atia \\ Qaiser, Orfeas Keramidas, Martin C Powell \\ Ambulatory Gynaecology Unit, Nottingham University \\ Hospitals, UK 2Chesterfield Royal Hospital, UK
}

Correspondence: Bee Fong Chen, Chesterfield Royal Hospital, Chesterfield Road, Calow, Chesterfield, S44 5BL Derbyshire, United Kingdom, Tel 44 776654I534,

Email beefongchen@hotmail.com

Received: June 15,2017 | Published: July 10, 2017
Abbreviations: EA, Endometrial Ablation; GA, General Anaesthetic; GnRH, Gonatrophin Releasing Hormone; HMB, Heavy Menstrual Bleeding; IUS, Intra-Uterine System; NICE, National Institute of Clinical Excellence; RCT, Randomized Controlled Trial; TRCE, Transcervical Resection of Residual Endometrium; TRCF, Transcervical Resection of Fibroid

\section{Introduction}

Second generation endometrial ablation (EA) is now a recognized non-invasive surgical method of treating heavy menstrual bleeding (HMB) after conservative medical treatments have failed. Thermal balloon EA is one of the main types of EA accepted by National Institute of Clinical Excellence (NICE) in the United Kingdom to be safe and effective in managing benign HMB. ${ }^{1,2} \mathrm{~A}$ further example of a second generation EAS is the bipolar radiofrequency, impedance controlled endometrial ablation system. ${ }^{3}$ These EA techniques require less surgical expertise and have been shown to have fewer complications as compared to first generation EA. ${ }^{4}$ Hence, they are more acceptable by patients to be performed as an outpatient procedure, which is quicker, simpler and low risk.

In our unit, the thermal balloon EA used is the Thermablate EAS. This lightweight device employs a reusable hand-held treatment control unit with a disposable fluid-filled balloon catheter system. An automatic treatment cycle of therapeutic high temperature $\left(110^{\circ} \mathrm{C}\right)$ involves inflation and deflation of the $13 \mathrm{ml}$ balloon catheter over 2.5 minutes. This causes uniform destruction of the endometrium with the employment of a pressure of $220 \mathrm{~mm} / \mathrm{Hg}$. ${ }^{5,6}$

The bipolar radiofrequency, impedance controlled endometrial ablation commonly used is the Novasure EA, it delivers radiofrequency energy until tissue impedance reaches $50 \mathrm{ohms}$ on average. The procedure is completed in approximately 90 seconds. ${ }^{6}$ The NovaSure EA has a proactive safety check prior to procedure to assess cavity integrity. If the integrity of the cavity is breached, the procedure is abandoned.

The literature suggests that both methods work well to produce amenorrhea and lighter periods and can successfully reduce the need for a hysterectomy, however radiofrequency ablation produces a higher rate of amenorrhea as compared to thermal ablation. ${ }^{4,7}$ However, repeat treatment or surgery is more likely after endometrial ablation in general as compared to hysterectomy. ${ }^{8}$ There are mixed conclusions regarding re-intervention and patient satisfaction within the different EA methods which is important as it may result in further distress and treatments. ${ }^{4,7}$ In our observational study, we have built a large database of both Thermablate EA and Novasure EA which have been used since 2008 in a tertiary teaching hospital.

\section{Materials and methods}

This study looked at the long-term intervention rates between July 2008 and July 2013 following Thermablate EA and NovaSure EA in 
women attending the Ambulatory Menstrual Disorders Clinic at the CIRCLE Treatment Centre in Nottingham. These women were all identified as having menorrhagia and had shown no previous response to conservative medical or hormonal therapy. The demographic differences between the two groups are demonstrated in Table 1. In addition they were counselled about reliable contraception and completion of family. The women were offered a choice of local or a general anaesthetic. 175 women had a Thermablate EA procedure and 133 women had a NovaSure ablation performed. 7 women in the Thermablate EA and 10 in the Novasure group were lost to follow-up. Both group of women had a median follow up of 19 months ranging from 6 to 60 months.

Table I Demographic differences between NovaSure 123 \& Thermablate 168

\begin{tabular}{|c|c|c|}
\hline Endometrial Ablation & NovaSure I 23 & Thermablate 168 \\
\hline \multicolumn{3}{|l|}{ BMI } \\
\hline $\mathrm{BMI} \leq 25$ & 35 & 32 \\
\hline BMI: $25-30$ & 44 & 51 \\
\hline $\mathrm{BMI} \geq 30$ & 43 & 85 \\
\hline BMI: Not recorded & 1 & 0 \\
\hline \multicolumn{3}{|l|}{ Mode of Delivery } \\
\hline P 0 & 6 & 8 \\
\hline $\mathrm{PI}$ & 12 & 13 \\
\hline Vaginal Delivery $\geq 2$ & 79 & 121 \\
\hline Caesarean Section & 17 & 19 \\
\hline Vaginal Delivery + Caesarean Section & 9 & 7 \\
\hline \multicolumn{3}{|l|}{ Ethnicity } \\
\hline White British & 103 & 143 \\
\hline White Asian/ Other Asian background & 1 & 1 \\
\hline Any other white/ mixed background & 6 & 4 \\
\hline Pakistani/ British Pakistani & 2 & 4 \\
\hline Caucasian & 1 & 1 \\
\hline Indian & I & 3 \\
\hline Black Caribbean & 1 & 3 \\
\hline Any other ethnicity & 8 & 9 \\
\hline
\end{tabular}

Initial diagnostic hysteroscopy identified eligible women as those having a normal uterine cavity and an endometrial biopsy was taken either prior to or just before the endometrial ablation. Women having malignant lesions or submucous fibroids bigger than 3 centimetres were excluded from the study. Some of the women received pretreatment prior to the endometrial ablation in the form of either the levonogestrel intra-uterine system (IUS) or hormone preparation (Norethisterone, Progesterone or Cerazette). The procedures were all conducted by the same surgeon.

In the ambulatory clinic, the women were advised to take oral analgesia in the form of 100 milligrams (mg) diclofenac and $50 \mathrm{mg}$ tramadol 2 hours preceding both methods of endometrial ablation. A perioperative intracervical block of $4 \%$ prilocaine and intracavitary 6 millilitres of $2 \%$ lidocaine gel were administered for LA. The women were discharged from the clinic post procedure usually within 30 minutes, provided they felt well and had achieved adequate pain relief.

The procedures carried out under general anaesthetic (GA) were all discharged home the same day, with a contact email address if they had any concerns or queries. The women received a telephone call the next day from the clinic nurse and were invited back to clinic for subsequent follow up at 4 months. Information for the study was gathered from 'NOTIS', the electronic system used within our Trust to hold patient data. Following the ablation subsequent procedures that occurred were listed, as well as the bleeding pattern recorded. As the data was collected anonymously without any patient details, ethical approval was not required.

The primary outcome of this study was to look at both the medical and surgical intervention rates following both types of endometrial ablation. We also explore patient satisfaction in terms of amenorrhea or better symptom control where there is an improvement in quality of life.

\section{Results}

123 women had the NovaSure procedure. 53 of the women had GA due to patient choice and where other procedures needed to be performed concurrently. 16 women had removal or replacement of an intrauterine system/device, and 3 women had concurrent insertion of IUS. 11 women had diagnostic laparoscopy, and 17 women had laparoscopic sterilization. 6 women had other more complex procedure concurrently such as laparoscopic management of ovarian cysts or endometriosis or insertion of a tension-free vaginal tape.

Although the 10 women who did not attend their follow-up appointment were not included and were not contactable, there were no hospital records of a further intervention received. 42 women were considered not to require outpatient follow-up after the NovaSure ablation due to excellent post-operative appearance. There were no reported recurrence of symptoms after the treatment and so no further interventions required. Of those who attended follow-up appointments, 16 women had lighter periods and 23 women had amenorrhea. Overall, 65 women stopped having periods including those who did not have appointments post operatively. However, 12 women $(9.7 \%)$ had described unacceptable bleeding at follow up and $17(13.8 \%)$ had cyclical pain despite being amenorrheic. 13 women (11\%) had symptoms of both pain and bleeding. Table 1 shows the comparison of symptoms post procedure in the two groups of women.

Overall 83 women $(67.5 \%)$ required no interventions following the NovaSure ablation (Figure 1). Of the women who needed nonsurgical additional treatments after the ablation, 8 had non-hormonal treatment i.e. counselling and analgesia. 7 women had hormonal management (Gonatrophin Releasing Hormone $(\mathrm{GnRH})$ analogues or Cerazette). 11 women $(8.8 \%)$ had further surgical interventions which excluded hysterectomy. These conservative surgical interventions included hysteroscopy, division of intra-uterine adhesions, removal of polyps, and transcervical resection of fibroid (TCRF)/transcervical resection of residual endometrium (TCRE). 23 women (18\%) had a hysterectomy for persistent symptoms of pain and bleeding whom had either failed to respond to further conservative management or requested to have this surgery.

In the Thermablate EA group of 175 women, although no hospital record could be found of a subsequent intervention, 7 patients were excluded who did not attend their follow-up appointment. 14 did not require follow up on a telephone enquiry.

Of the women who had Thermablate EA, 43 women (25.6\%) had amenorrhea and 81 women (48.2.\%) had lighter menstrual periods. 3 women had symptoms of pelvic pain. 33 women had unacceptable bleeding (19.6\%) and 6 women (3.6\%) had both on-going bleeding and pelvic pain. 2 women had post-operative infection, one of which developed a haematometra. The comparison of symptoms between the two types of endometrial ablation is shown in Table 1. 127 women $(75.6 \%)$ had no interventions following Thermablate EA (Figure 1). 4 women $(2.2 \%)$ had non hormonal conservative treatment. 7 women 
(4\%) had medical treatment in the form of oral norethisterone and GnRH analogues. 31 women had conservative surgical interventions which in the main were TCRF and/or TCRE. 14 (8\%) women in the Thermablate EA went on to have hysterectomy for persistent uterine bleeding.

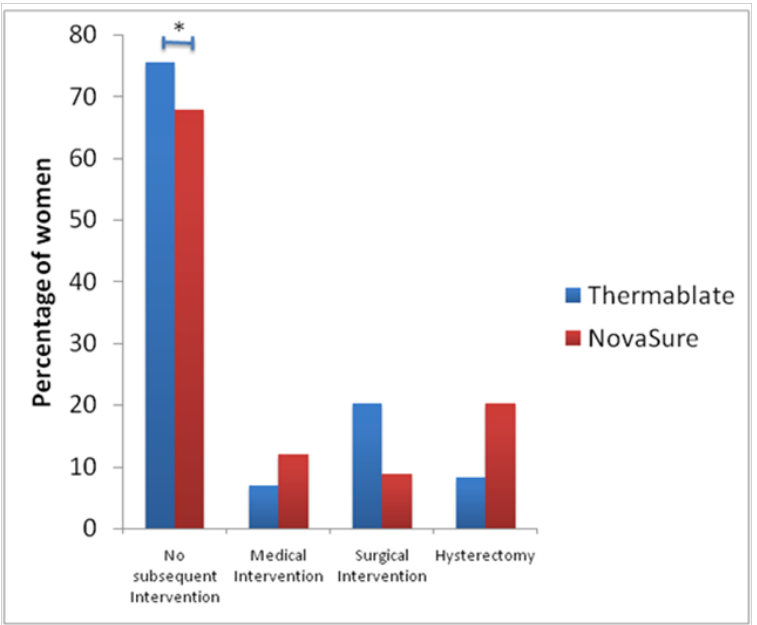

Figure I The percentages of women needing intervention after Thermablate and Novasure ablation system.

The blue bars represent percentage of women who underwent Thermablate ablation whilst the red bars represent women in the Novasure group.

Asterisk indicates a statistical difference in the no subsequent interventions group between the two types of ablations $(p=0.001)$ where $67.5 \%$ in the Novasure group as compared to the Thermablate ablation of $75.6 \%$.

In relation to interventions post procedure, there is a statistical difference between the two types of ablations $(p=0.001)$ where $67.5 \%$ in the Novasure group with no subsequent interventions as compared to the Thermablate EA group of $75.6 \%$. In terms of medical management, $6.55 \%$ of Thermablate EA group had non-surgical treatments, which were both non-hormonal and hormonal interventions as compared to $12.2 \%$ of the Novasure patients. However, surgical conservative interventions were more common in the Thermablate EA group. Hysteroscopic resection of the residual endometrium was performed in $11(8 \%)$ of NovaSure patients as compared to $31(17.7 \%)$ of the Thermablate. More importantly, the odds ratio of women having hysterectomy in the Novasure as compared to Thermablate EA group is 2.8 with $95 \%$ confidence interval of 1.37 and 5.71 (Table 2).

Table 2 Symptoms experienced after Thermablate and Novasure endometrial ablation system

\begin{tabular}{|c|c|c|}
\hline Symptoms & Thermablate EAS & NovaSure System \\
\hline Amenorrhea & $25.6 \%$ & $63.4 \%$ \\
\hline Satisfied, Lighter Bleeding & $48.2 \%$ & $13 \%$ \\
\hline Cyclical Pain & $1.8 \%$ & $13.8 \%$ \\
\hline On-going Bleeding & $19.6 \%$ & $9.7 \%$ \\
\hline On-going bleeding and pain & $3.6 \%$ & $11 \%$ \\
\hline Infection post procedure & $1.2 \%$ & $0 \%$ \\
\hline
\end{tabular}

EAS: Endometrial Ablation System

\section{Discussion}

In this study, the high rate of amenorrhea with the Novasure system is consistent with previous studies compared to the
Thermablate group., ${ }^{4,9}$ Although if we stated that the combined symptoms of amenorrhea and a lighter menstrual period concluded a satisfied patient, the two groups had comparable rate. In most studies, amenorrhea is considered a successful primary outcome but another important outcome to consider is the subsequent re-intervention rate $^{7,8}$ This has implications on women's morbidity and mortality who require further intervention to control their symptoms. It might have been predicted that as in this study, as more patients become amenorrheic with the NovaSure system, the re-intervention rate in this group would have been lower. However in our study, the Thermablate EA patients had a lower rate of hysterectomy compared with the NovaSure group.

This could be due to the mechanism of the Novasure EA destroying more of the uterine cavity and endometrial thickness than the Thermablate causing the higher rate of amenorrhea. The destruction of the endometrial lining may result in adhesions of the uterine walls obscuring the cavity in an Asherman's like scenario. This makes conservative secondary interventions such as an endometrial resection not possible. The results of this process are seen in the group of patients with persistent symptoms of cyclical pelvic pain, with or without on-going bleeding after a NovaSure. A hysterectomy may then be the only available next option. A network meta-analysis in 2012 looked at bipolar radiofrequency ablation, microwave ablation, balloon ablation (Thermachoice) and free fluid ablation. ${ }^{4}$ They found at 12-month follow up there was a higher rate of amenorrhea in those who had had bipolar radiofrequency or microwave ablation compared to thermal ablation but there was no convincing evidence between the three techniques in the number of women who were dissatisfied or still experiencing heavy menstrual bleeding. In a randomized controlled trial (RCT) of 65 women, Penninx et al. ${ }^{7}$ explored the 5 -year outcomes following bipolar radiofrequency impedance-controlled ablation (NovaSure) and hydrothermablation (Hydro Thermablator System). ${ }^{7}$ As compared to our study, the amenorrhea rate is similar at $63.4 \%$ and theirs at $55 \%$ for Novasure and $28.6 \%$ and $35.4 \%$ for thermal ablation. Surgical re-intervention is similar in the Novasure group with $11 \%$ in Penninx et al. ${ }^{7}$ study and $17.7 \%$ in our study. However, their thermal ablation re-intervention is higher at $35.4 \%$ as compared to our study at $8 \%$.

In one of the latest RCT which was a continuation from 5-year follow by Penninx et al. ${ }^{7} \&$ Herman et al. ${ }^{10}$ demonstrated at ten years after treatment, the superiority of bipolar ablation over balloon ablation in the treatment of heavy menstrual bleeding was no longer evident. ${ }^{10}$ In our study, it has shown Thermablate EAS is likely a better 2nd generation endometrial ablation as compared to the NovaSure system. The overall patient's satisfaction rates are similar but a lower hysterectomy rate. The Thermablate EA has additional advantages in it being simpler to use and ideal for an ambulatory gynecology unit.

We appreciate our study is an observational study and would benefit from a randomized controlled trial design. Nonetheless, we managed to collect data from a large number of women which represented the population in our area.

\section{Conclusion}

In conclusion, our study showed thermal ablation has lower longterm re-intervention rate as compared to Novasure system and reduced rate of hysterectomy. Women should be given an informed choice especially for women who wish to conserve their uterus. In addition this has important cost implication. We feel that the benefits of an endometrial thermal ablation system that is well tolerated, perfect for an outpatient scenario. 


\section{Acknowledgements}

We would like to thank Dr Eu-Wing Toh from Leeds Institute of Molecular Medicine, Leeds for his advice on statistical analyses and Dr George Vilos from the department of Gynecology at the University of Western Ontario, Canada for his support and advice on writing the paper.

\section{Conflicts of Interest}

None

\section{References}

1. National Institute for Health and Clinical Excellence. Heavy menstrual bleeding: assessment and management. Clinical Guideline No. 44. London, UK. 2007.

2. National Institute of Health and Clinical Excellence. Fluid filled therma balloon and microwave endometrial ablation techniques for heavy menstrual bleeding, London, UK. 2004.

3. Cooper J, Gimpelson RJ. Summary of safety and effectiveness data from FDA: a valuable source of information on the performance of global endometrial ablation devices. J Reprod Med. 2004;49(4):267-273.

4. Daniels JP, Middleton LJ, Champaneria R, et al. Second generation endometrial ablation techniques for heavy menstrual bleeding: network meta-analysis. BMJ. 2012;344:e2564.

5. Karamanidis D, Nicolaou P, Byros A, et al. Two-year results of a new two-minute hot liquid balloon endometrial ablation system (Thermablate): a pilot study. Clin Exp Obstet Gynecol. 2009;36(4):256258

6. Cooper J, Gimpelson RJ. Summary of safety and effectiveness data from FDA: a valuable source of information on the performance of global endometrial ablation devices. J Reprod Med. 2004;49(4):267-273.

7. Penninx, JPM, Herman, MC, Mol BW, et al. Five-year followup after comparing bipolar endometrial ablation with hydrothermablation for menorrhagia. Obstet Gynecol. 2011;116(4):819-826.

8. Lethaby A, Shepperd S, Cooke I, et al. Endometrial resection and ablation versus hysterectomy for heavy menstrual bleeding. Cochrane Database Syst Rev. 1999;2:CD000329.

9. Gallinat A. NovaSure impedance controlled system for endometrial ablation: three-year follow-up on 107 patients. Am J Obstet Gynecol. 2004;191(5):1585-1589.

10. Herman MC, Penninx JPM, Mol BW, et al. Ten-year followup of a randomized controlled trial comparing bipolar endometrial ablation with balloon ablation for heavy menstrual bleeding. BJOG. 2013;120(8):966970 